\title{
Random Walk Behaviour of Malaysia Share Return in Different Economic Circumstance
}

\author{
${ }^{1}$ Muhammad Fadhil Marsani* and ${ }^{2}$ Ani Shabri \\ ${ }^{1}$ School of Mathematical Sciences, Universiti Sains Malaysia \\ 11800 Minden, Penang, Malaysia \\ ${ }^{2}$ Department of Mathematical Sciences, Faculty of Science, Universiti Teknologi Malaysia \\ 81310, UTM Johor Bahru, Johor, Malaysia \\ ${ }^{*}$ Corresponding author: fadhilmarsani@gmail.com
}

Article history

Received: 30 September 2018

Received in revised form: 7 May 2019

Accepted: 10 July 2019

Published online: 1 December 2019

\begin{abstract}
This journal renders the random walk behaviour of the Malaysian daily share return, through tests of efficient market hypothesis (EMH) based on three different financial periods, namely growth, financial crisis, and recovery period. This review also covers the behaviour of extreme return for weekly and monthly series generated from Block maxima-minima method. Autocorrelation Function test (ACF) and Ljung-Box test had been employed to measure average correlation between observations, while Augmented Dickey-Fuller (ADF), Phillips-Perron (PP), Kwiatkowski Phillips Schmidt Shin (KPSS) test had been used to scan the unit root and the stationarity. Multiple variance ratio tests had also been conducted to examine the random walk behaviour. Serial correlation test indicated that the movement of daily return during the financial crisis period was weak-form efficiency. The unit root and stationary tests suggested that each daily series was stationary, but trend stationary for extreme cases. Variance ratio tests indicated that the return during the recovery period was weak-form inefficiency due to the short lag autocorrelation in series.
\end{abstract}

Keywords Extreme share returns; Block maxima method; Non-stationary; Random walk; KLCI.

Mathematics Subject Classification 05C81, 60G50, 62-07, 62G32

\section{Introduction}

The random walk theory is commonly used in defining the presence of erratic movements in series. A critical aspect of random walk in time series is the series are random and unpredictable. Examining stationarity and random walk behaviour in share returns is crucial for investors to gain precise calculation and establish proper risk management, since occurrence of random walk in time series analysis could lead to a spurious and unreliable result[1],[2]. 
The random walk behaviour in share returns was pioneered by [1], who then proposed the concept of the Efficient market hypothesis (EMH). This concept is essentially an investment idea in which share prices correspond to all available information. EMH suggests that the movement of the share price is of weak-form efficiency if the price demonstrates unpredictable action upon reflecting all appropriate information and random walk designating the return. On the contrary, the prices are of weak-form inefficiency when the series is likely to be projected by the past returns because of no randomness effect. The idea of random walks in share prices has significant consequences in finance and investment theories. Governing authorities have to ensure a share market function as a random walk so that equity is at balance level and properly valued at efficient capital allocation.

This research concerns with the random walk pattern in Malaysian share market. Previously, analyses on Malaysian stock market efficiency demonstrated mixed finding in rejecting the EMH (see[3],[4],[5],[6],[7]). The rationale of the present study is to confirm the movement of the Malaysian financial markets by considering the economic circumstance in daily return. This research also covers inspection on the behaviour of random walk and stationary condition in extreme share return for the weekly and monthly intervals.

\section{Methodology}

\subsection{Data}

KLCI daily stock returns of the period from Jan 1994 to June 2008 (14 years) were taken from Yahoo Finance. For different economic circumstance analysis, data sample had been split into three economic environments, namely growth (Jan 1994- June 1997), crisis (July 1997Dec 2001), and recovery (Jan 2002- June 2008) period. These partitions were set according to Malaysian quarterly gross domestic product growth report. Share return was computed using $R_{t}=\ln \left(P_{t} / P_{t-1}\right)$, where $R_{t}$ is return index at $t$ time, $P_{t}$ is share price index in terms of $t$, while $P_{t-1}$ is share price index at time $t-1$.

\subsection{Extreme Value Theory and Extreme Risk Modelling}

In extreme value theory, two primary methods are generally applied, namely the block maximaminima method (BMM), and the peaks over threshold (POT) method. In this paper, the focus is only on BMM because this approach is unrivaled in demonstrating extreme share price volatility for a given interval. Among researchers who also used this method in their analysis are $[8],[9],[10]$. In a study conducted by [11], it was found that whenever the returns were independent and identically distributed (i.i.d), an extreme limiting distribution had to be the Generalized Extreme Value Distribution (GEV).

\subsection{Block Maxima Method and Generalized Extreme Value Distribution (GEV)}

Under the block maxima approach, the extremes are defined as the maximum data point in successive periods. [12] acknowledged that the limiting distribution of these extremes is the generalized extreme value (GEV) distribution.

The block maxima approach considers maximum variable takes in successive periods. For example, let $X_{1}, X_{2}, \ldots, X_{n}$ be a sequence of independent, identically distributed (i.i.d) random 
variables with a standard distribution function $F(x)=P\left(X_{i} \leq x\right)$, which does not have to be known. The block maxima approach requires grouping the series into non-overlapping successive blocks and identifying the maximum from each block, as $M_{n}=\max \left(X_{1}, \ldots, X_{n}\right)$. The limit law of the block maxima is given by the Fisher-Tippett theorem, as: Let $\left(X_{n}\right)$ be a sequence of (i.i.d) random variables. If there exist series of constants $c_{n}>0, d_{n} \in R$ and some non-degenerate distribution function, $H$ such that

$$
\frac{M_{n}-d_{n}}{c_{n}} \stackrel{d}{\longrightarrow} H \text { as } n \rightarrow \infty
$$

then, $\mathrm{H}$ belongs to one of the three standard extreme value distributions:

$$
\begin{aligned}
& \text { Frechet }: \Phi_{\alpha}(x)= \begin{cases}0, & x \leq 0 \\
e^{-(x)^{-\alpha}}, & x>0, \alpha>0\end{cases} \\
& \text { Weibull }: \psi_{\alpha}(x)= \begin{cases}e^{-(-x)^{-\alpha}}, & x \leq 0, \alpha>0 \\
1, & x>0\end{cases} \\
& \text { Gumbel }: \Lambda(x)=e^{-e^{-x}, x \in R}
\end{aligned}
$$

Collectively, these three families of distribution are termed as the extreme value distributions. Each family has location, $d$, and scale parameter, $c$. Additionally, the Fréchet and Weibull families have a shape parameter $\alpha$. Parameter $\alpha$ is the tail index, and indicates the thickness of the tail of the distribution; thicker tail means smaller tail index. The beauty of this theorem is that these three distributions are the only possible limits of the distribution of the extremes $M_{n}$, regardless of the distribution, $F$ of the population. In this sense, this theorem provides an extreme value version of the central limit theorem.

[13] noted that extreme return could be the most minimum or the most maximum of a stock index, according to the given interval period; in our study, it refers to weekly and monthly periods. For weekly and monthly intervals, the maximum and minimum series can be set as chosen blocks of 5 and 20 trading days for weekly and monthly, respectively. This approach can be written in terms of mathematical equations as:

$$
\begin{aligned}
& x_{1}=\max \left(R_{1}, R_{2}, \ldots, R_{m}\right), \\
& x_{2}=\max \left(R_{m+1}, R_{m+2}, \ldots, R_{2 m}\right), \ldots, \\
& x_{n / m}=\max \left(R_{n-m}, R_{n-m+1}, \ldots, R_{n}\right)
\end{aligned}
$$

where $R_{1}, R_{2}, \ldots, R_{n}$ are daily share returns, $n$ denotes total sample observation, and $m$ is the size of the timespan block.

\subsection{Random Walk Hypothesis}

$[1],[2],[14]$ were one of the first to study the random walk, and who pointed out that the random walk model can be measured by using three successively restrictive hypotheses criteria: firstly(RW1), the return is serially uncorrelated but dependent; secondly(RW2), returns are serially uncorrelated, independent but not identically distributed; and thirdly (RW3), returns are serially uncorrelated and identically distributed. In this analysis, serial dependence was inspected using Ljung box and Autocorrelation Function (ACF) plot tests to answer RW1, 
while stationarity condition was observed using Augmented Dickey-Fuller (ADF), PhillipsPerron (PP), and Kwiatkowski Phillips Schmidt Shin (KPSS) tests to answer RW2. Lastly, distributional assumptions were checked using multiple variance ratio tests; specifically LoMacKilay test, to answer RW3.

Random walk is used to define the changes in the price index that are independent of each other. A random walk with drift process can be written as:

$$
X_{t}=X_{t-1}+\delta+\varepsilon_{t}
$$

where $X_{t}$ is the log of the price at time $t, \delta$ is a drift constant, $\varepsilon_{t}$ is a random disturbance term with $E\left(\varepsilon_{t}\right)=0, \sigma_{\varepsilon}^{2}$ is constant, and $E\left(\varepsilon_{t} \varepsilon_{t-\tau}\right)=0$ for $\tau \neq 0$. Drift constant $(\delta)$ is a weighted average of the possibilities of the share price could move to the subsequent point.

\subsection{Serial Correlation}

Serial correlation, among other series, is observed to sense the existence of the random walk. A random walk is presumed when the returns are uncorrelated at all lags with no serial correlation in the series. The serial correlation coefficient $(\rho)$ of share returns at lag $k$ can be written as

$$
\rho(k)=\frac{\sum_{t=1}^{n-k}\left(R_{t}-R_{\mu}\right)\left(R_{t-k}-R_{\mu}\right)}{\sum_{t=1}^{n}\left(R_{t}-R_{\mu}\right)^{2}}
$$

where $R_{t}$ is the share return for a period, $t, R_{t-k}$ signifies returns over the period, and $R_{\mu}$ as the mean return. Correlation coefficient, $\rho_{k}$, may give negative or positive values, indicating negative or positive serial correlation, or even zero correlation which denotes random movement of the series. An autocorrelation function $(\mathrm{ACF})$ is a graphical representation of serial correlation coefficient which displays correlation structure in different values of lag.

\subsection{Ljung-Box Test}

Ljung-Box test is conducted to examine autocorrelations when a group of a time series is nonzero. By this test, the randomness can be inspected for overall and each number of lags. The hypotheses for Ljung-Box testcan be expressed as:

$H_{0}$ : The data are independently distributed (randomness in the series) vs

$H_{1}$ : The data are not independently distributed (serial correlation is present) where Ljung-Box statistic is given as

$$
Q_{L B}=n(n+2) \sum_{k=1}^{h} \frac{\hat{\rho}_{k}^{2}}{n-k}
$$

where $\hat{\rho}_{k}$ denotes correlation coefficient at lag, $k, h$ denotes the number of tested lags, and $n$ denotes the sample size (refer to [15] for clearer view about this test). 


\subsection{Unit Root Test}

Augmented Dickey-Fuller (ADF) test is applied for determining the existence of a unit root in a time series. Ordinary Least Squares regression (OLS) is then operated to estimate the unknown parameter, as in the following equation:

$$
\Delta R_{t}=\alpha_{0}+\alpha_{1} t+\rho_{0} R_{t-1}+\sum_{i=1}^{k} \rho_{i} \Delta R_{i t-i}+\varepsilon_{i t}
$$

where $R_{t}$ denotes share return at time t, $\Delta R_{t}$ denotes a difference in share return represented by $R_{t}-R_{t-1}, k$ denotes the number of lagged terms, $\alpha_{i}$ denotes the trend coefficient, $\alpha_{0}$ denotes a constant, t denotes the time, $\rho_{i}$ denotes the estimating coefficients, and $\varepsilon$ denotes the white noise. Similar to the Augmented Dickey-Fuller test, The Phillips-Perron (PP) test consolidates as an alternative nonparametric technique in restraining the serial correlation. PP test checks a unit root by using non-augmented Dickey-Fuller equation and revising the test statistic to assure that the asymptotic distribution is unchanged by serial correlation.

The hypotheses for both of this test is,

$$
\begin{aligned}
& H_{0} \text { : unit root is present in the series vs } \\
& H_{1} \text { : the series is either stationary or trend-stationary } \\
& \quad \text { (stationary around a deterministic trend) }
\end{aligned}
$$

The time series has the possessions of a random walk if the test fails to reject $H_{0}$ (refer to [18] and [19] for more details about this test).

\subsection{KPSS Test}

In this study, to examine if the apparent sample was a non-stationary or deterministic trend, we employed Kwiatkowski Phillips Schmidt Shin (KPSS) tests. The model for random walk, stationary random error and sum of a deterministic trend can be written as

$$
\begin{aligned}
& y_{t}=d_{t}+r_{t}+\varepsilon_{t}, \\
& r_{t}=r_{t-1}+u_{t},
\end{aligned}
$$

where $d_{t}=\sum_{i=0}^{p} \beta_{i} t^{i}$, for $p=0,1$ comprises of the constant or deterministic trend, $r_{t}$ signifies a random walk with variance $\sigma_{u}^{2}, u_{t}$ distributed as IID $N\left(0, \sigma_{u}^{2}\right)$, while $\varepsilon_{t}$ is distributed as IID $N\left(0, \sigma_{\varepsilon}^{2}\right)$. The test statistic is given as:

$$
L M=\sum_{t=1}^{T} \frac{s_{t}^{2}}{\hat{\sigma}_{\varepsilon}^{2}}
$$

in which the KPSS test hypothesis for a random walk with zero variance was based on LM test. The hypothesis for this test was expressed as

$$
\begin{aligned}
& H_{0} \text { : the series is stationary or trend-stationary }\left(\sigma_{u}^{2}=0\right) \text { vs } \\
& H_{1} \text { : the series has a unit root and non-stationary }\left(\sigma_{u}^{2}>0\right)
\end{aligned}
$$

If the test fails to reject $H_{0}$, there is presence of randomness in the sample series (refer to [18] for clearer view about this test). 


\subsection{Lo-MacKilay Test}

The purpose of Lo-MacKilay test is to explore whether or not a sample behaves like a random walk. The test statistic $M_{1}(k)$ is given by

$$
M_{1}(k)=\frac{V R(x ; k)-1}{\phi(k)^{0.5}}
$$

where $k$ is the period return, $\operatorname{VR}(x ; k)$ is the asymptotic distribution by assuming that $k$ is fixed when $T \rightarrow \infty, \phi(k)$ is the asymptotic variance, which can be written as

$$
\phi(k)=\frac{2(2 k-1)(k-1)}{3 k T}
$$

The conditional heteroscedasticity in $x_{t}$ is adapted by the robust statistic for heteroscedasticity $M_{2}(k)$, given as

$$
M_{2}(k)=\frac{V R(x ; k)-1}{\phi(k)^{0.5}}
$$

where the wild bootstrap is implemented to $M_{2}(k)$. The hypotheses for both Lo-MacKilay tests can be described as

$$
\begin{aligned}
& H_{0} \text { : the series follows the random walk vs } \\
& H_{1} \text { : the series does not follow the random walk }
\end{aligned}
$$

The time series behaves as the random walk if the test fails to reject $H_{0}$. More information regarding this test can be referred from [19],[20].

\section{Results and Discussion}

This section discusses the experimental results of this study. The first highlight is the KLCI daily price movement, followed by descriptive statistics and reflection on the effects on the stationarity and random walk.

Figure 1 depicts three different movements in the KLCI daily share price from 1994 to 2008. Growth period (Jan 1994 to June 1997) is marked by a green line, while crisis period (July 1997 to Dec 2001) is characterised by a red line, and recovery period (Jan 2002 to June 2008) is marked by a blue line. Here, daily share price and economic transition shifted along with periodical arrangements.

Initialy, the price index continued to fluctuate around 1200 points before it reached a critical stage, when the index dipped to the lowest at 200. In the recovery phase, as expected, the price index steadily increased, reaching peak at 1600 before it reduced slightly to 1200 . Figure 2 displays the daily KLCI price in terms of log returns. Generally, the value was between -0.1 and 0.1. The volatiles of the KLCI return were much more significant during the crisis time interval, and once the daily log returned, it fluctuated in range of -0.2 and 0.2 .

Table 1 displays the descriptive statistics for daily (overall, growth, crisis and recovery), extreme weekly, and extreme monthly returns. In this analysis, standard deviation was applied to measure the dispersion of the data series, while skewness was used to measure distribution asymmetries, and kurtosis was utilized to size the fat-tail of the probability distribution, besides 


\section{KLSE Price 1994-2008}

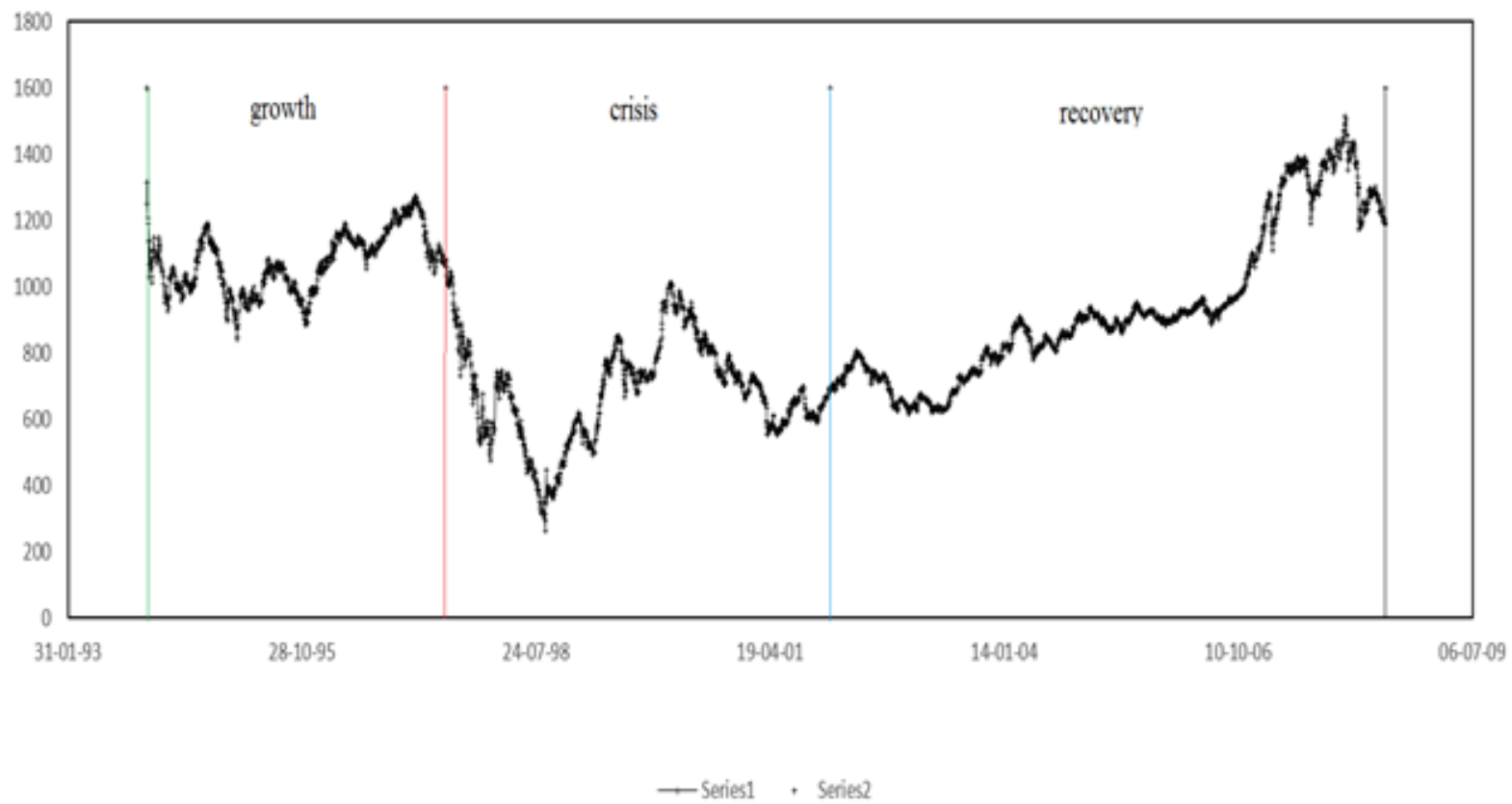

Figure 1: KLCI Daily Share Price

applying Jarque-Bera test (JB) to calculate the normality of the data dispersions. Interestingly, the highest (0.2082) and lowest (-0.2415) returns were recorded during the crisis period. This period produced the highest value of standard deviation $(2.4190 \%)$, skewness $(0.5023)$, and kurtosis (25.0443), indicating higher risk possibility during this crisis period compared to other periods.Among skewness of daily interval, the recovery period was the only interval which recorded a negative skewness (-1.3966) with the tail inclined to the left, indicating a higher probability of gaining a positive return.

In terms of extreme weekly and monthly returns, generally, the mean and skewness for the extreme maximum interval showed positive values, indicating that extreme maximum range had a positive performance, but otherwise for the extreme minimum. Each of the series did not show suitable approximate by normal distribution because of the large Jb statistics and significant p-value.

Figure 3 presents the price return and ACF plot for KLCI daily share return, namely overall, growth, crisis, and recovery period. The trends in time series plot are shown as red lines. ACF plots of each of the periods exhibited relatively quick decay to zero, especially during crisis period, where the ACF plot was immediately cut off after the first lag, indicating lack of serial correlation among the observation. For the recovery period, the ACF plots were only cut off after the fourth lag, meaning there was slight autocorrelation in the series. Figure 4 depicts the price return and ACF plot for extreme (maximum and minimum) weekly and monthly intervals. The suggestive trends line in price return plot indicates the existence of serial correlation and nonstationary. ACF plot displayed slow decline, signifying that the series had a high level of 
autocorrelation, and the sequence was not random.

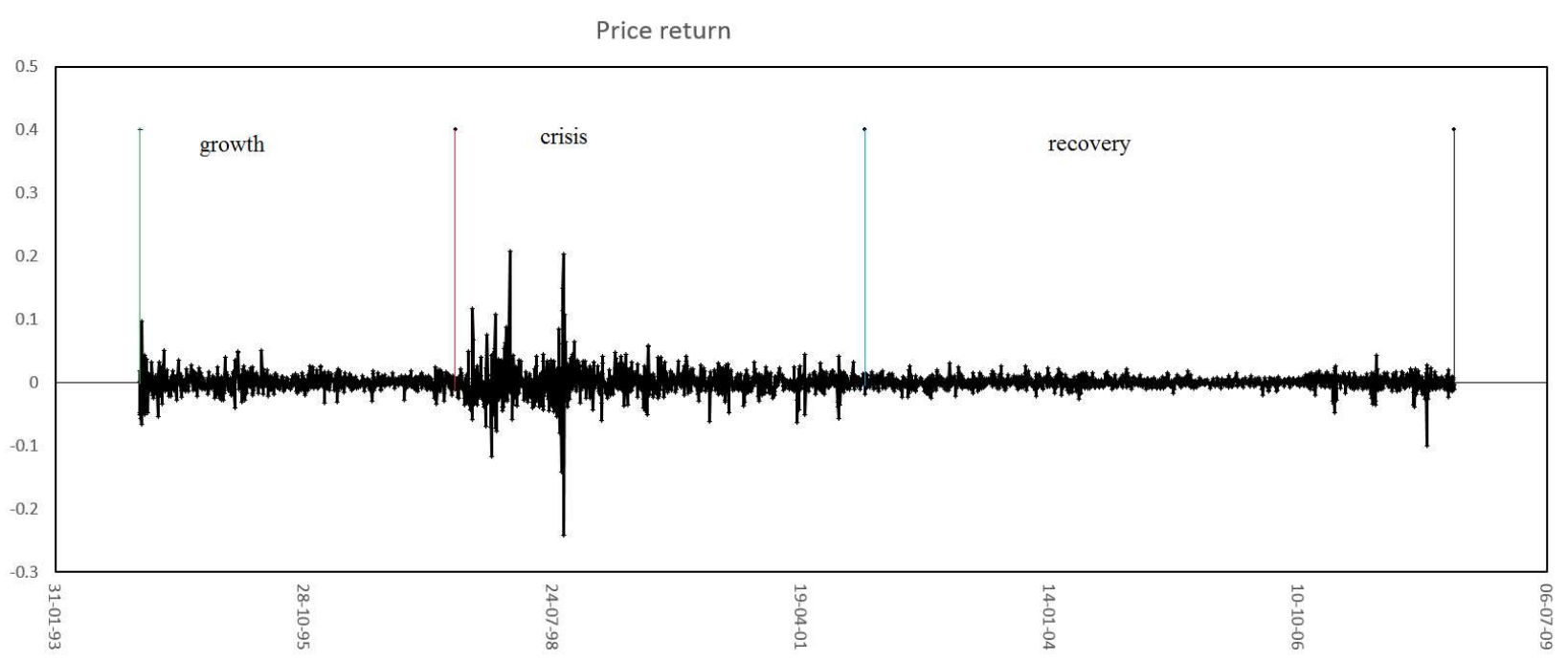

Figure 2: KLCI Daily Price Log Return

Table 2 presents the serial correlation and unit root test. Box-Ljung (3) test showed that serial correlation existed in each of the intervals, except for daily crisis interval when the p-value failed to reject $\mathrm{H} 0$ of no serial correlation, indicating weak-form efficiency (unpredictable) during this period. In all cases for Box-Ljung (20), the H0 was rejected, meaning that at higher lag, the series exhibited serial correlation. Meanwhile, for unit root test, Augmented Dickey-Fuller (ADF) and Phillips Perron (PP) tests had been used to check the existence of unit root, while Kwiatkowski-Phillips-Schmidt-Shin (KPSS) test had been used to inspect the stationarity. The results for unit root test using $\mathrm{ADF}$ and $\mathrm{PP}$ implied rejection of $\mathrm{H} 0$ in all intervals, suggesting there was no random movement in daily and extreme share return. Next, KPSS test was conducted to confirm the stationarity. In this test, $\mathrm{H} 0$ was rejected at all intervals, except in extreme cases, indicating that the series for extreme return is non-stationary.

Next, further investigation using random walk test had been conducted to confirm the series behaviour. Table 3 presents the result of Lo-MacKilay test, under random walk test. In this test, the focus was only on $\mathrm{M} 2(\mathrm{k})$ as the aim was to examine for possible heteroscedasticity.

Note that, the holding periods $(\mathrm{k})$ considered for Lo-MacKilay test were $\mathrm{k}=2,5,10$ and 30. The simulations had been carried out by setting up the number of bootstrap iterations $\mathrm{m}$ to 1000 . The $2.5 \%$ and $97.50 \%$ represent the lower and upper limit of confidence intervals for Lo-Mackinlay tests from Bootstrap distribution. This test indicated insignificant p-value for all of the daily cases, except for recovery period, indicating that return during the recovery period did not follow a random walk. In summary, these results have shown that the daily returns of overall, growth, and crisis series were weak-form efficiency (unpredictable) and followed a random walk. This confirms that the daily recovery and extreme returns for the weekly and monthly intervals did not behave as a random walk and autocorrelation existed in the series.

\section{Conclusion}

This paper has reviewed investigation on stationarity behaviour and random walk process of daily and extreme return for weekly and monthly series generated from Block maxima-minima 

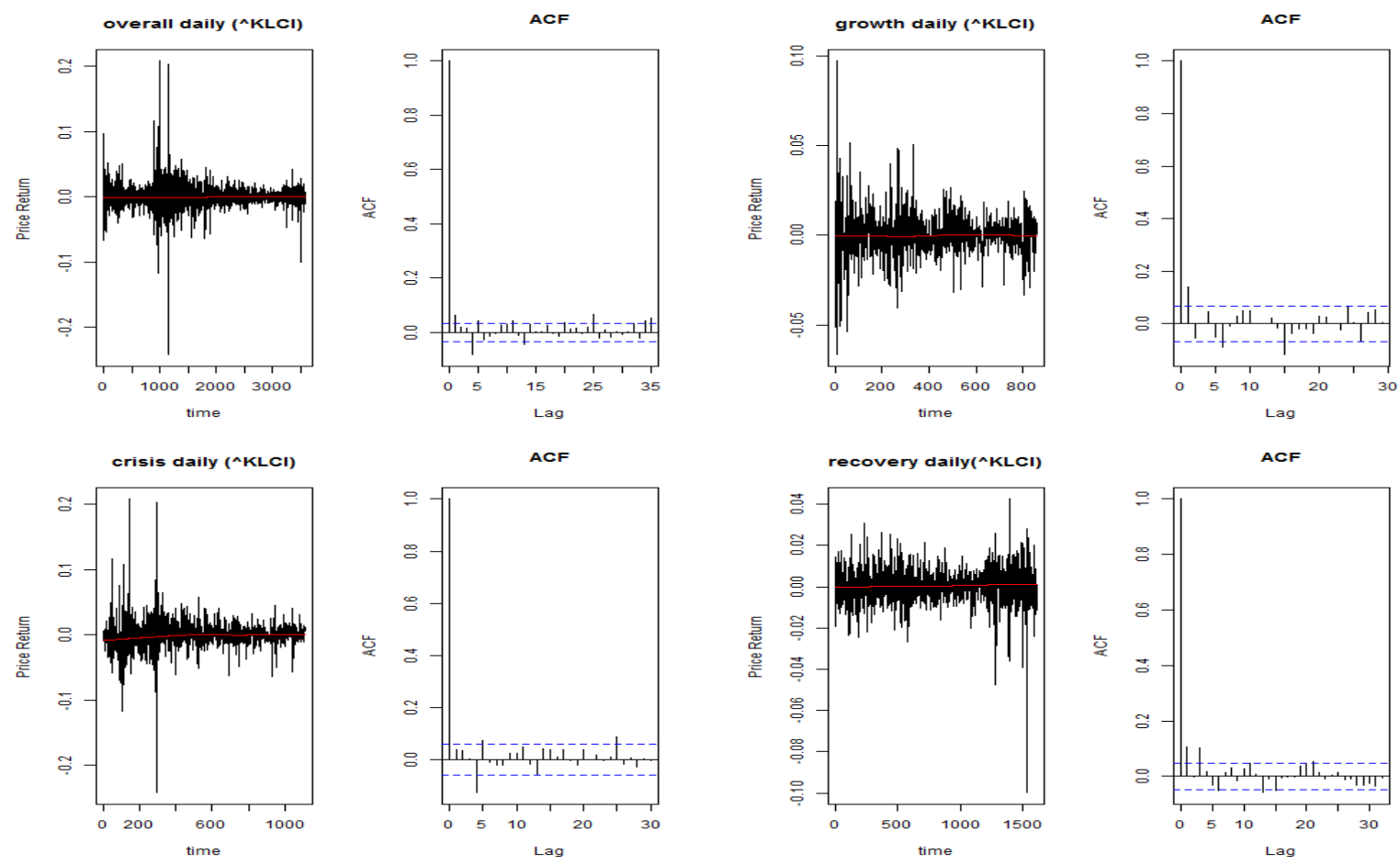

Figure 3: KLCI Daily Price Log Returns
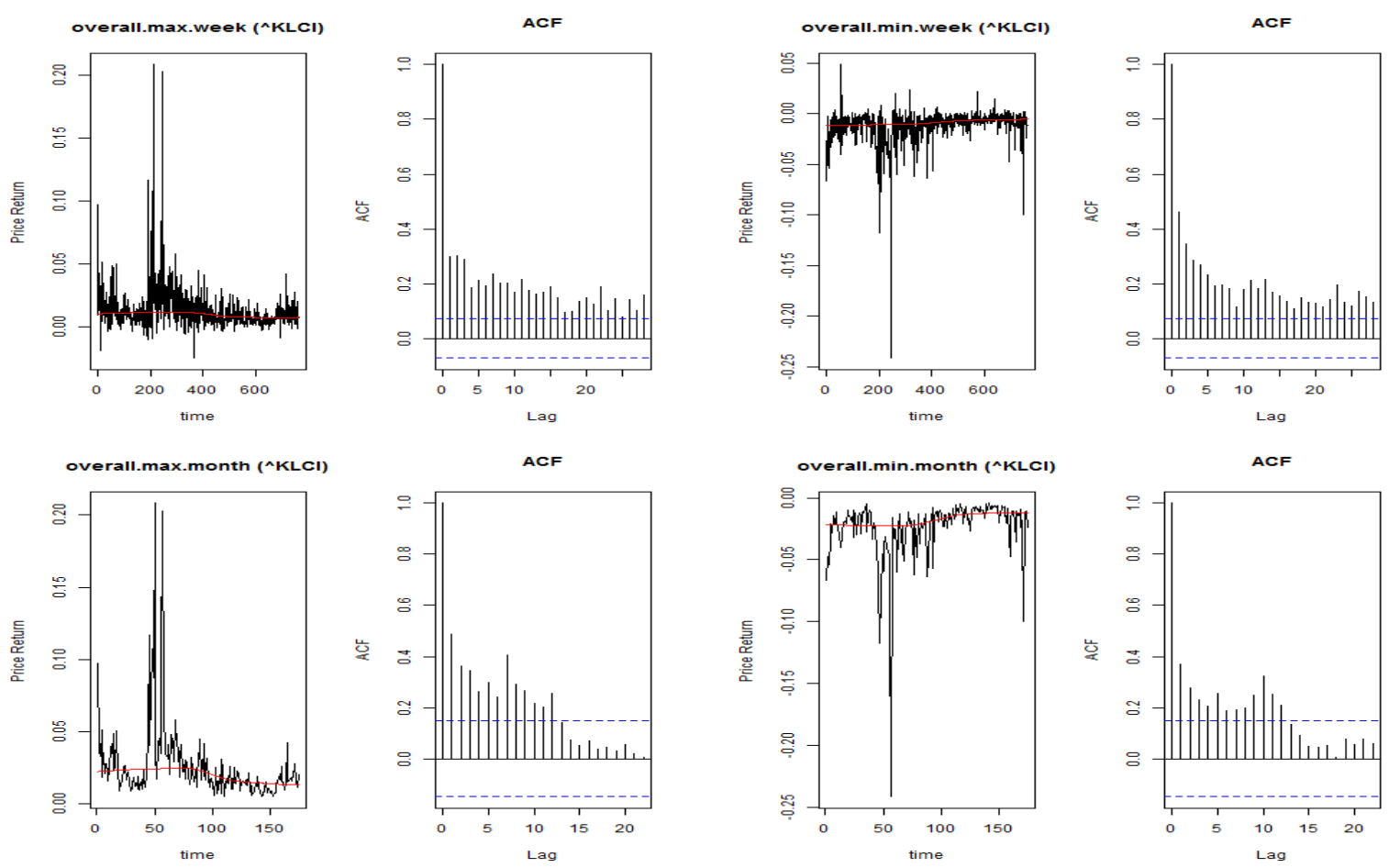

Figure 4: KLCI Daily Price Log Returns 
Table 1: Descriptive Statistics

\begin{tabular}{|l|c|c|c|c|c|c|c|c|c|}
\hline Interval & obs & mean(\%) & max & min & sd(\%) & skewness & kurtosis & Jb.test & pval \\
\hline daily.overall & 3577 & -0.0023 & 0.2082 & -0.2415 & 1.5817 & 0.4454 & 44.0272 & 250989.4000 & 0.0000 \\
\hline daily.growth & 859 & -0.0209 & 0.0971 & -0.0665 & 1.2629 & 0.2067 & 10.2635 & 1894.4200 & 0.0000 \\
\hline daily.crisis & 1110 & -0.0399 & $\mathbf{0 . 2 0 8 2}$ & $\mathbf{- 0 . 2 4 1 5}$ & $\mathbf{2 . 4 1 9 0}$ & $\mathbf{0 . 5 0 2 3}$ & $\mathbf{2 5 . 0 4 4 3}$ & 22521.9100 & 0.0000 \\
\hline daily.recovery & 1608 & 0.0336 & 0.0426 & -0.0998 & 0.8216 & -1.3966 & 19.0579 & 17799.0000 & 0.0000 \\
\hline extreme.max.week & 761 & 1.3347 & 0.2082 & -0.0243 & 1.6968 & 5.3712 & 50.9778 & 76647.5300 & 0.0000 \\
\hline extreme.min.week & 761 & -1.2449 & 0.0486 & -0.2415 & 1.6941 & -5.0528 & 54.3198 & 86749.1700 & 0.0000 \\
\hline extreme.max.month & 174 & 2.5828 & 0.2082 & 0.0051 & 2.6437 & 4.3195 & 26.9295 & 4692.5990 & 0.0000 \\
\hline extreme.min.month & 174 & -2.4117 & -0.0043 & -0.2415 & 2.4556 & -4.7848 & 38.1414 & 9617.0810 & 0.0000 \\
\hline
\end{tabular}

Table 2: Serial Correlation and Unit Root Test

\begin{tabular}{|l|c|c|c|c|c|c|c|c|c|c|}
\hline Interval & BL (3) & pval & BL (20) & pval & ADF & pval & PP & pval & KPSS & pval \\
\hline daily.overall & 17.0375 & 0.0007 & 81.6143 & 0.0000 & -14.7081 & 0.0100 & -3288.6159 & 0.0100 & 0.2140 & 0.1000 \\
\hline daily.growth & 19.1997 & 0.0002 & 51.6861 & 0.0001 & -9.1035 & 0.0100 & -708.7192 & 0.0100 & 0.1499 & 0.1000 \\
\hline daily.crisis & $\mathbf{3 . 4 1 5 0}$ & $\mathbf{0 . 3 3 2 0}$ & 43.8694 & 0.0016 & -9.4227 & 0.0100 & -1049.3004 & 0.0100 & 0.2239 & 0.1000 \\
\hline daily.recovery & 35.9975 & 0.0000 & 65.3344 & 0.0000 & -10.6180 & 0.0100 & -1490.5764 & 0.0100 & 0.0827 & 0.1000 \\
\hline extreme.max.week & 201.5720 & 0.0000 & 615.1371 & 0.0000 & -5.4455 & 0.0100 & -726.9304 & 0.0100 & $\mathbf{2 . 0 6 8 4}$ & $\mathbf{0 . 0 1 0 0}$ \\
\hline extreme.min.week & 315.7409 & 0.0000 & 738.6583 & 0.0000 & -5.9626 & 0.0100 & -501.2113 & 0.0100 & $\mathbf{1 . 7 2 1 9}$ & $\mathbf{0 . 0 1 0 0}$ \\
\hline extreme.max.month & 86.5098 & 0.0000 & 220.9169 & 0.0000 & -3.4603 & 0.0481 & -106.2196 & 0.0100 & $\mathbf{1 . 0 5 8 4}$ & $\mathbf{0 . 0 1 0 0}$ \\
\hline extreme.min.month & 47.5926 & 0.0000 & 146.7132 & 0.0000 & -3.4640 & 0.0478 & -125.5994 & 0.0100 & $\mathbf{0 . 8 9 5 4}$ & $\mathbf{0 . 0 1 0 0}$ \\
\hline
\end{tabular}

BL(3), BL(20) Ljung-Box third order and twentieth serial correlation in the residuals

Table 3: Bootstrap Variance Ratio Tests

\begin{tabular}{|c|c|c|c|c|c|c|c|c|c|c|c|c|}
\hline Interval & \multicolumn{3}{|c|}{$K=2$} & \multicolumn{3}{|c|}{$\mathrm{K}=5$} & \multicolumn{3}{|c|}{$\mathrm{K}=10$} & \multicolumn{3}{|c|}{$\mathrm{K}=\mathbf{3 0}$} \\
\hline & $2.50 \%$ & $97.50 \%$ & p.val & $2.50 \%$ & $97.50 \%$ & p.val & $2.50 \%$ & $97.50 \%$ & p.val & $2.50 \%$ & $97.50 \%$ & p.val \\
\hline daily.overall & -1.8066 & 1.7532 & 0.4790 & -1.5796 & 2.1171 & 0.5150 & -1.4781 & 2.2982 & 0.6900 & -1.4591 & 2.4693 & 0.4380 \\
\hline daily.growth & -1.9325 & 1.8681 & 0.0130 & -1.8199 & 2.0952 & 0.1890 & -1.7321 & 2.2298 & \begin{tabular}{|l|}
0.5650 \\
\end{tabular} & -1.5152 & 2.3078 & 0.9240 \\
\hline daily.crisis & -1.7617 & 1.8235 & 0.7460 & -1.5197 & 2.1901 & 0.7780 & -1.3891 & 2.3292 & 0.9110 & -1.4222 & 2.4821 & 0.5960 \\
\hline daily.recovery & -1.8983 & 1.9089 & 0.0050 & -1.7764 & 2.0518 & 0.0040 & -1.7587 & 2.0921 & 0.0330 & -1.7533 & 2.1333 & 0.0620 \\
\hline extreme.max.week & -1.8150 & 1.7807 & 0.0000 & -1.6845 & 1.9345 & 0.0000 & -1.6296 & 2.1204 & 0.0000 & -1.4541 & 2.2283 & 0.0000 \\
\hline extreme.min.week & -1.7815 & 1.7574 & 0.0000 & -1.5787 & 2.0227 & 0.0000 & -1.5485 & 2.2138 & 0.0000 & -1.4247 & 2.3987 & 0.0000 \\
\hline extreme.max.month & -1.8677 & 1.8492 & 0.0000 & -1.5971 & 2.0540 & 0.0000 & -1.4995 & 1.9689 & 0.0000 & -1.2365 & 1.8841 & 0.0000 \\
\hline extreme.min.month & -1.8061 & 1.7079 & 0.0010 & -1.6764 & 1.9533 & 0.0000 & -1.6628 & 1.8866 & 0.0000 & -1.4005 & 1.5489 & 0.0000 \\
\hline
\end{tabular}

method. Random walks in daily and extreme returns had been studied by investigating several different criteria, specifically the parametric serial correlation (RW1), stationarity condition (RW2), and distributional assumptions (RW3). Ljung-box test and ACF plot had been employed to examine RW1, while Augmented Dickey Fuller, Phillips Perron and Kwiatkowski Phillips Schmidt Shin tests had been applied to examine RW2, besides the use of Variance Ratio Tests to test RW3.

The first contribution of this study is we have inspected the existence of nonstationary in Malaysian extreme share return. The extreme series was predictable, with evidence of rejection of random walk hypotheses in weekly and monthly extreme intervals. The second contribution is the use of economic factor in the analysis, which is evident by overall daily (Jan 1994 to June 2008), growth (Jan 1994 to June 1997), and crisis (July 1997 to Dec 2001); indicating that the return period was weak-form efficiency and followed a random walk. Nevertheless, during 
recovery period (Jan 2002 to June 2008), the series does not weak-form efficiency due to the existence of autocorrelation in returns. Also, daily return during crisis period had higher loss risk possibility compared to others. Overall, this study strengthens the notion of the need for non-stationary method idea in extreme return analysis in future investigations.

\section{Acknowledgments}

The authors would like to thank Universiti Sains Malaysia and the Ministry of Higher Education for the grant of scholarship.

\section{References}

[1] Fama, E. F. Efficient capital markets: a review of theory and empirical work. The Journal of Finance. 1970. 25(2): 383-417.

[2] Fama, E. F. Efficient capital markets: II. The Journal of Finance. 1991. 46(5): 1575-1617.

[3] Mun, F. W. and Kee, K. S. Do Asian stock market prices follow martingales? Evidence from spectral shape tests. Asia Pacific journal of Management. 1994. 11(2): 345-359.

[4] Kawakatsu, H. and Morey, M. R. An empirical examination of financial liberalization and the efficiency of emerging market stock prices. Journal of Financial Research. 1999. 22(4): 385-411.

[5] Mohamad, S. The Efficiency of the Kuala Lumpur Stock Exchange: A Collection of Empirical Findings. Penerbit Universiti Pertanian Malaysia: 1993.

[6] Hoque, H. A. A. B., Kim, J. H. and Pyun, C. S. A comparison of variance ratio tests of random walk: A case of Asian emerging stock markets. International Review of Economics E Finance. 2007. 16(4): 488-502.

[7] Kim, J. H. and Shamsuddin, A. Are Asian stock markets efficient? Evidence from new multiple variance ratio tests. Journal of Empirical Finance. 2008. 15(3): 518-532.

[8] Hussain, S. I. and Li, S. Modeling the distribution of extreme returns in the Chinese stock market. Journal of International Financial Markets, Institutions and Money. 2015. 34: 263-276.

[9] Marsani, M. F. , Shabri, A., and Jan, N. A. M. Examine generalized lambda distribution fitting performance: An application to extreme share return in Malaysia. Malaysian Journal of Fundamental and Applied Sciences. 2017. 13(3): 230-237

[10] Tolikas, K. Unexpected tails in risk measurement: Some international evidence. Journal of Banking and Finance. 2014. 40(1): 476-493.

[11] Gnedenko, B. Sur la distribution limite du terme maximum d'une serie aleatoire. Annals of Mathematics. 1943. 1(1): 423-453.

[12] Fisher, R. A. and Tippett, L. H. C. In Limiting Forms of the Frequency Distribution of the Large Sample. Cambridge University Press. 1928.

[13] Longin, F. M. The asymptotic distribution of extreme stock market returns. Journal of Business. 1996. 69(3): 383-408. 
[14] Campbell, J. Y., Campbell, J. W., Lo, A. W.-C., MacKinlay, A. C., Champbell, J. J., LO, A. A. and Lo, A. W. The Econometrics of Financial Markets. Princeton University press: 1997.

[15] Box, G. E. P. and Pierce, D. A. Distribution of residual autocorrelations in autoregressiveintegrated moving average time series models. Journal of the American statistical Association. 1970. 65(332): 1509-1526.

[16] Said, S. E. and Dickey, D. A. Testing for unit roots in autoregressive-moving average models of unknown order. Biometrika. 1984. 71(3): 599-607.

[17] Perron, P. Trends and random walks in macroeconomic time series: Further evidence from a new approach. Journal of Economic Dynamics and Control. 1988. 12(2-3): 297-332.

[18] Kwiatkowski, D., Phillips, P. C. B., Schmidt, P. and Shin, Y. Testing the null hypothesis of stationarity against the alternative of a unit root: How sure are we that economic time series have a unit root? Journal of Econometrics. 1992. 54(1-3): 159-178.

[19] Kim, J. H. Wild bootstrapping variance ratio tests. Economics Letters. 2006. 92(1): 38-43.

[20] Lo, A. W. and MacKinlay, A. C. Stock market prices do not follow random walks: Evidence from a simple specification test. The Review of Financial Studies. 1988. 1(1): 41-66. 